\title{
Walther Heissig
}

(5 décembre 1913 - 5 septembre 2005)

\section{Françoise Aubin}

\section{(2) OpenEdition \\ Journals}

Édition électronique

URL : https://journals.openedition.org/emscat/1015

DOI : 10.4000/emscat.1015

ISSN : 2101-0013

Éditeur

Centre d'Etudes Mongoles \& Sibériennes / École Pratique des Hautes Études

\section{Édition imprimée}

Date de publication : 1 mai 2006

Pagination : 465-469

ISBN : 2-9518888-3-X

ISSN : 0766-5075

Référence électronique

Françoise Aubin, «Walther Heissig », Études mongoles et sibériennes, centrasiatiques et tibétaines [En ligne], 36-37 | 2006, mis en ligne le 24 février 2009, consulté le 13 juillet 2021. URL : http://

journals.openedition.org/emscat/1015; DOI : https://doi.org/10.4000/emscat.1015

Ce document a été généré automatiquement le 13 juillet 2021.

(c) Tous droits réservés 


\section{Walther Heissig}

(5 décembre 1913 - 5 septembre 2005)

\section{Françoise Aubin}

Walther Heissig, sur le seuil de son bureau, avec sa femme Annemarie, dans leur maison de Rheinböllen en octobre 2001

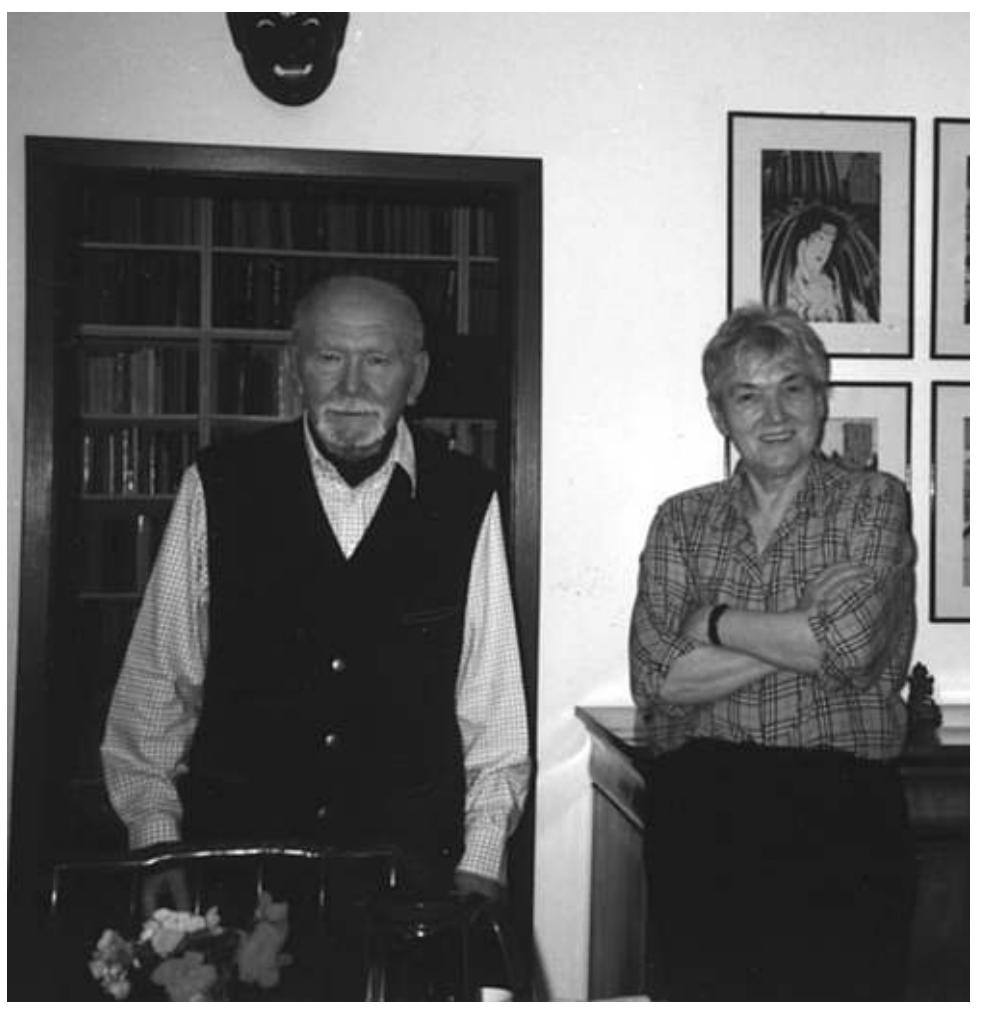

Françoise Aubin

1 Voici brusquement les mongolisants devenus orphelins: le lundi 5 septembre 2005, s'éteignait le Maître dont l'œuvre protéiforme a exploré pratiquement tous les champs possibles des études mongoles (si l'on met à part la linguistique, dominée entre autres par son aîné, Nicholas Poppe, 1897-1991), un savant dont la bienveillante cordialité 
semblait trouver réponse à toutes les questions que pouvaient lui adresser ses jeunes collègues. Heureusement pour la postérité, ce fut un travailleur acharné comme il en existe peu, qui a publié sans discontinuer, depuis les années quarante et presque jusqu'au terme de sa longue vie, articles et livres à remplir des étagères entières, où les mongolisants des générations futures puiseront sources textuelles et inspiration. Il n'y a qu'à voir comment sa bibliographie se gonfle entre l'hommage qui lui est rendu pour ses soixante-dix ans (Michael Weiers dans Documenta Barbarorum, Wiesbaden: Otto Harrassowitz, 1983, en allemand, pp. XI-XXXII), celui de ses quatre-vingts ans (D. Cerensodnom, dans Mongolica, Ulaanbaatar, 4, $\mathrm{n}^{\circ}$ 25, 1993, en mongol, pp. 5-28), enfin celui de ses quatre-vingt-dix ans (par Udo B. Barkmann, dans Acta Mongolica, Ulaanbaatar, T. 3, n 215, 2004, en allemand, pp. 7-32).

2 Né à Vienne en 1913, dans la toute fin de l'ambiance cosmopolite de la capitale de l'empire austro-hongrois, il a terminé ses jours quatre-vingt onze ans plus tard dans la campagne de Rheinböllen, sur le plateau de Hunsrück dominant la vallée du Rhin, à une quarantaine de kilomètres au sud de Koblenz, dans un charmant cottage d'où émanait, pour le visiteur étranger, un parfum indubitablement autrichien.

3 Il paraît que c'est la lecture de Sven Hedin qui l'a, tout jeune, attiré vers les études orientales. En 1936 (il a alors 23 ans), il part pour Berlin, étudier le chinois et le mongol auprès du grand Eric Haenisch (1880-1966), le fondateur de l'étude moderne de Saghang Sechen et l'auteur de la première transcription et de la première traduction en Occident de l'Histoire secrète, un homme d'une intégrité morale exemplaire en ce temps de montée du national-socialisme. W. Heissig, lui, ne peut échapper à un intermède militaire en 1940 ; néanmoins, il réussit en 1941 - on se demande par quel miracle d'énergie et d'intelligence - à être diplômé à Vienne en études mongoles, sinologiques, ethnologiques et préhistoriques, à défendre une thèse, récompensée d'un summa cum laude, sur les changements culturels dans la province du Hsingan (Xing'an) au Manchoukuo (Manzhouguo), d'après des sources mongoles contemporaines - Der mongolische Kulturwandel in den Hsingan-Provinzen Mandschukuos (thèse parue, avec des remaniements et des compléments, en 1944, Wien-Pékin : Siebenberg-Verlag), à publier son premier livre (Das gelbe Vorfeld. Die Mobilisierung der chinesischen Aussenländer, Heidelberg: Vowinckel, 163 p) ; à collaborer, avec R. Bleichsteiner, à la rédaction d'un manuel du mongol contemporain sous forme d'un synopsis grammatical et d'un lexique, sous la direction de W.A. Unkrig (Wörterbuch der heutigen mongolischen Sprache, Wien-Pékin : Siebenberg-Verlag, 136 p.)

Cette même année 1941 voit son départ pour la Mongolie-Intérieure et la Mandchourie, certes à titre militaire, ce qui lui a été reproché en 1945, mais avait-il alors un autre choix ? De 1943 à 1946, il enseigne le mongol à Pékin, à la Faculté de philosophie de l'Université catholique Fu-jen, tenue par la congrégation catholique allemande de Steyl (Pères du Verbe Divin, SVD) et il est en contact fréquent avec le P. Antoine Mostaert, CICM, l'inventeur de la dialectologie mongole. Ses publications scientifiques commencent bientôt à le signaler lui-même comme l'initiateur d'une nouvelle voie d'approche de la réalité mongole, tandis que les possibilités de séjour dans les zones mongoles, qui lui ont été ouvertes durant l'occupation japonaise, lui ont permis d'enrichir prodigieusement sa connaissance de la littérature mongole orale et écrite. Ici, se situe une petite énigme historique : un périodique en langue anglaise publié sous l'égide de l'occupant japonais, Contemporary Manchuria, propose dans son numéro d'octobre 1939 (III, nº 4) un article de Walther Heissig, « Mongol Farming », qui semble 
ignoré de ses biographes jusqu'à nos jours, on ne sait pourquoi. Il s'agit là d'une enquête très documentée menée dans un village du Hsing-an, donc un travail réalisé après l'arrivée de l'auteur sur le terrain: la date précoce de cette parution, qui semblerait avoir précédé tous ses autres travaux connus, provient sans doute d'un retard dans la publication du volume; et le titre est, sans contredit, à ajouter à la bibliographie du Maître.

En 1951, on le retrouve enseignant le mongol à l'université de Göttingen, où il fonde, en 1954, en collaboration avec Siegfried Lienhard et Omeljan Pritsak, une collection de monographies dite Göttinger Asiatische Forschungen : bien que celle-ci projette de couvrir «l'histoire, les langues et la littérature des peuples de l'Asie méridionale, orientale et centrale ", neuf des dix volumes parus avant sa clôture en 1958 sont concentrés sur l'histoire et la littérature des seuls peuples mongols (ainsi en est-il notamment du $\mathrm{n}^{\circ} 2$, Die Pekinger lamaistischen Blockdrucke in mongolischer Sprache, de W. Heissig, 1954, et, en

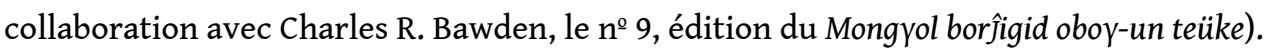

6 En 1957, il transfère ses talents à l'Université de Bonn (fondée en 1818 par FrédéricGuillaume III de Prusse). Et, en 1964, créant là ce qui allait très rapidement devenir le grand centre européen d'études littéraires et historiques mongolo-tibétaines, le Seminar fur Sprach und Kulturwissenschaften Zentralasiens, il trouve une tribune à la hauteur de ses capacités. Jusqu'à l'âge de la retraite et de l'éméritat, en 1982, il a dirigé cette institution, splendidement installée dans les «mansardes » de la loge de la résidence à Bonn du prince électoral et archevêque de Cologne, en style baroque ; et, par la suite, il a continué à y faire de fréquentes visites, s'intéressant à son avenir, malgré la centaine de kilomètres qui le séparait de Bonn.

7 Tous les indices qui permettent de qualifier un maître se concentrent sur lui. Les disciples d'abord: les maîtres de la génération suivante, qui vont s'illustrer dans les études mongolo-tibétaines et au-delà, ont été ses élèves, ainsi Klaus Sagaster, le premier à avoir soutenu sa thèse de doctorat sous sa direction et le premier à lui avoir succédé à la chaire de l'Asie centrale, Michael Weiers, Rudolph Kaschewsky, HansRainer Kämpfe, Veronika Veit, etc.; et d'autres encore ont pu mener leurs recherches grâce à un ambitieux Projet de recherche spéciale, interdisciplinaire, le Sonderforschungsbereich, qu'il a fondé en 1969 et qu'il a longtemps dirigé. Et on lui doit bien d'autres créations fécondes, telle la Permanent International Altaistic Conference en 1957, en collaboration avec Denis Sinor et Annemarie von Gabain - une P.I.A.C. qui a connu, l'été 2005, sa $48 \S$ session, cette fois-ci près de Moscou; telle la collection de monographies héritière des Göttinger Asiatische Forschungen, les Asiatische Forschungen à partir de 1959, riches en 2005 de 148 volumes (publiés par la maison d'édition Otto Harrassowitz à Wiesbaden), au sein desquels des sous-collections se détachent, ainsi les volumes du Folklore mongol de l'érudit mongol B. Rintchen et ceux des Mongolischen Epen (dont les deux premiers volumes sont une traduction des tomes I et II de Rintchen); tel le solide périodique ZentralasiatischeStudien dont il a été le rédacteur en chef du numéro 1 en 1975 au numéro 31 en 2001 (le numéro 33 est sorti en 2004) et qui a fourni une floraison exceptionnelle d'articles d'érudition; des entreprises telles que des symposiums internationaux sur l'épopée qui ont permis de réunir autour d'une même table des savants venus de l'Ouest et de l'Est, de République populaire de Mongolie et de République populaire de Chine et de publier leurs articles dans une collection spéciale.

Un maitre, il l'a été aussi par l'ampleur des champs qu'il a couverts et l'étendue des horizons qu'il a dégagés. A lui, revient le mérite d'avoir sorti les études mongoles du 
ghetto chinois et du carcan philologique dans lesquels l'érudition extrême-orientaliste de la première moitié $d u x^{e}$ siècle avait tendance à les enfermer. Non qu'il ait nié, cela va de soi, les influences littéraires chinoises, présentes, par exemple, dans le genre du bensen üliger, auquel est consacré un de ses derniers gros travaux (Si Liyang, 'Asiatische Forschungen', Bd 131, 1996), mais il a amplement démontré que la culture littéraire mongole existait en elle-même, hors du monde sinophone. Non qu'il ait manifesté, non plus, le moindre laxisme dans l'exactitude de ses traductions, mais les arguties sur l'étymologie des termes et sur l'éventuelle parenté des langues dites altaïques ne l'intéressaient pas. Sa tâche s'est fixée plus haut : grâce à lui, une abondante littérature mongole historique, poétique, romancée ou religieuse est entrée dans le patrimoine culturel mondial, ce qui fut une découverte, non seulement pour les comparatistes, mais pour les mongolisants aussi.

Le travail fondamental de défrichage a été le catalogage et l'identification des textes mongols (ainsi que des cartes territoriales) disponibles en imprimés parfois, plus souvent en xylographes ou en manuscrits à travers le monde, puis l'édition d'une partie d'entre eux, leur commentaire, leur traduction au moins partielle. Son histoire de la littérature mongole des $\mathrm{xIX}^{\mathrm{e}}$-xxe siècles, en deux gros volumes (Geschichte der mongolischen Literatur, Harrassowitz, 1972), est restée un monument, qui force l'admiration par la richesse de ses sources et la maestria avec laquelle sont décelés et classés les courants et tendances de la littérature écrite et orale des Mongols depuis deux siècles.

Le rôle de l'église bouddhique est visiblement important dans le développement de cette littérature, fournissant d'une part des textes traduits du tibétain et des motifs exemplaires propres à inspirer les contes didactiques, d'autre part des lieux de haute éducation : c'est cette influence qui intéresse Walther Heissig, notamment en ce qu'elle a modelé le chamanisme tardif et les rites populaires. Deux volumes, rééditant dans les années quatre-vingt-dix une vingtaine d'articles précédents de 1944 à 1992, témoignent que le chamanisme et les rites sont bien deux domaines de recherche qui l'ont passionné toute sa vie (dans Schamanen und Geisterbeschwörerin der östlichen Mongolei. Gesammelte Aufsätze, Harrassowitz, 1992, on remarquera, pp. 61-135, un gros article qui a fait sensation en son temps, "A Mongolian source to the Lamaist suppression of Shamanism in the 17th century ", jadis publié dans Anthropos, 48, 1953; et dans Götter im Wandel. Gesammelte Aufsätze zum Synkretismus der mongolischen Volksreligion, Harrassowitz, 1996, sont regroupés, par exemple, des invocations à divers Ciels et au Vieillard blanc, des textes de rituels de purification ou de consécration). Alors que, de nos jours, la Mongolie post-communiste se recrée une tradition de rites religieux et para-religieux identitaires, et que les jeunes mongolisants - ceux qui sont formés en France notamment - sont attirés par l'enquête ethnographique, précieuse reste la leçon qu'a dispensée Walther Heissig tout au long de sa longue vie créative : à savoir l'importance des textes et de leur traduction dans la description des rites et des coutumes, l'enquête factuelle sur le terrain étant complémentaire - ne l'a t'il d'ailleurs pas pratiquée lui aussi, dans ses débuts, durant son séjour au Hsing-an? On peut citer, en exemple typique de la manière de faire particulière à Walther Heissig, un séduisant article, avec photos, décrivant la bibliothèque d'un minuscule monastère du Tsakhar, en Mongolie-Intérieure : ce sont 61 volumes en mongol et 21 en tibétain de xylographes pékinois datant des dernières décennies du xVIIß siècle et des premières du xVIII ${ }^{e}$, maintenant conservés au Musée historique de Berne, dont il lit et traduit les colophons 
et dont il examine les implications historiques («Eine kleine mongolische Klosterbibliothek aus Tsakhar ", Jahrbuch des Bernischen Historischen Museums in Bern, XLI-XLII, 1961-1962, pp.557-590).

11 Enfin les deux passions qui se sont affirmées chez lui au fil des ans ont été pour l'épopée - en attestent les colloques et les publications qu'il a patronnés sur ce thème - et, très fidèlement jusqu'à la fin, pour le conte populaire mêlé de poésie. Aussi longtemps que sa vue le lui a permis, il s'est distrait en lisant les contes et histoires publiés en Mongolie-Intérieure dans l'écriture mongole traditionnelle, et son œuvre ultime, parue l'année de son quatre-vingt-dixième anniversaire, est une analyse des motifs et de leurs diverses variantes dans les collections de folklore mongol parus en Chine depuis une vingtaine d'années (Motive und Analysen mongolischer Märchen, 'As. Forsch.', Bd 146, 2003).

12 La liste des honneurs qui ont rendu hommage à son oeuvre et à son action est longue : membre correspondant de la Societas Orientalis Fennica dès 1952 et, en 1960, de la Société finno-ougrienne, membre de la Nordrhein-Westfälische Akademie der Wissenschaften (en 1967), puis de la Rheinisch-Westfälische Akademie der Wissenschaften, de la Société Kőrösi Csoma à Budapest (en 1976), de la Bayerischen Akademie des Wissenschaften à Munich (en 1980), de la Societas Uralo-Altaica à Göttingen (en 1981), de la British Academy (en 1988), de l'Académie des Sciences de Mongolie à l'époque du post-communisme (en 1994); il a été aussi le récipiendaire de la médaille d'or de la PIAC en 1982, et, en 2003, du prix d'honneur de l'International Association for Mongol Studies à Ulaanbaatar. Il était également Honorary Fellow de la SOAS de Londres, professeur honoraire de l'Université d'Ulaanbaatar et de celle de Hohhot; enfin, en 2003, il recevait en Mongolie le plus haut honneur, sous la forme de la décoration de "l'Étoile polaire " (Altan gadas). Mais il faudra sans doute encore longtemps pour que soit prise la pleine et totale mesure de son apport aux études mongoles et au comparatisme de la littérature orale. 